\title{
Complutum
}

ISSN: 1131-6993

\section{Presente y futuro de las técnicas aplicadas al estudio de la estacionalidad}

\author{
Antonio Jesús Sánchez Flores ${ }^{1}$; Mari Carmen Arriaza ${ }^{2}$; José Yravedra Sainz de los Terreros ${ }^{3}$
}

Recibido: 23 de enero de 2018 / Aceptado: 26 de noviembre de 2018

Resumen. A mediados del siglo XX, los métodos de los estudios de estacionalidad surgieron para intentar establecer el momento del año y el tiempo de ocupación en el que se habitaron los yacimientos arqueológicos. Sin embargo, las limitaciones de estas técnicas, basadas fundamentalmente en restos óseos fósiles, han dificultado la interpretación de estos datos. Este trabajo hace una relación de la mayoría de métodos utilizados para los estudios estacionales hasta la fecha, para facilitar su comprensión y su futura aplicación, destacando las virtudes y los defectos de cada uno.

Palabras clave: Estacionalidad; Zooarqueología; Dientes; Yacimientos arqueológicos.

\section{[en] Present and future of the techniques applied to seasonality studies}

Abstract. In the mid-twentieth century, the methods of seasonality studies emerged to try to establish the time of year, and the occupation time, in which the archaeological sites were inhabited. However, the limitations of these techniques, based fundamentally on fossil skeletal remains, have hindered the interpretation of these data. This work makes a list of most methods used for seasonal studies so far, in order to facilitate their understanding and application in the future, highlighting the virtues and shortcomings of each.

Keywords: Seasonality; Zooarchaeology; Teeth; Archaeological sites.

Sumario: 1. Introducción. 2. Métodos y técnicas aplicadas a los estudios de estacionalidad. 2.1. Métodos indirectos. 2.2. Métodos directos. 2.2.1. Estudios de estacionalidad en peces y moluscos. 2.2.2. Estudios de estacionalidad en mamíferos y aves. 2.3. Métodos directos basados en dientes. 2.3.1. Erupción y desgaste dental. 2.3.2. Microdesgaste. 2.3.3. Cementocronología. 2.3.4. Análisis de isótopos. 3. Discusión. 4. Conclusiones.

Cómo citar: Sánchez Flores, A.J.; Arriaza, M.C.; Yravedra Sainz de los Terreros, J. (2018): Presente y futuro de las técnicas aplicadas al estudio de la estacionalidad. Complutum, 29(2): 407-426.

\section{Introducción}

Algunas de las preguntas que surgen al estudiar un yacimiento arqueológico es precisar en qué momento y durante cuánto tiempo fueron habitadas. Las ocupaciones de un yacimiento pueden producirse durante un evento singular o durante largos periodos de tiempo. Esto se puede responder a través de la geoarqueología (Polo et al. 2016; Carrancho et al. 2016), la tafonomía (Yravedra et al. 2016a) o la arqueoestratigrafía (Spagnolo et al. 2016; Bargalló et al. 2016), además de la estacionalidad (Rivals et al. 2009a, 2009b; Rivals y Semprebon 2012; Sánchez-Hernández et al. 2014; Julien et al. 2015; Rodríguez-Hidalgo et al. 2016).

\footnotetext{
Universidad Complutense de Madrid. Departamento de Prehistoria antsan04@ucm.es

2 University of the Witwatersrand. School of Geography, Archaeology and Environmental Studies. Centre of Excellence in Palaeosciences, University of Witwatersrand, Johannesburg, South Africa. maria.arriazadorado@wits.ac.za

3 Universidad Complutense de Madrid. Departamento de Prehistoria jyravedr@ghis.ucm.es
} 
Sin embargo, el momento del año en el que se ocupó un yacimiento solo puede responderse a través de los análisis de estacionalidad. Estos análisis pueden precisar las relaciones estacionales entre diferentes yacimientos ( $\mathrm{Ri}$ vals y Deniaux 2005; Rivals y Solounias 2007; Rivals y Semprebon 2012), o las estrategias cinegéticas especializadas sobre determinados recursos (Monks 1981; Rodríguez-Hidalgo et al. 2016, 2017); o estrategias cinegéticas diferenciales entre recursos (Pike-Tay et al. 1999; Arceredillo y Díez 2009; Rivals et al. 2009a; Rendu 2010), e incluso, podrían llegar a precisar momentos de agregación por motivos simbólicos, como los potlach o la caza comunal (Monks 1981; Rodríguez-Hidalgo et al. 2017 y referencias en el mismo).

Aunque los estudios de estacionalidad pueden resultar de gran utilidad para la interpretación de los yacimientos, su análisis también entraña una gran dificultad. Frecuentemente, la falta de comprensión de la biología y del comportamiento de las especies estudiadas, junto con condicionantes medioambientales, sedimentarios o tafonómicos, pueden llevar a aplicaciones metodológicas erróneas e interpretaciones discutibles (Milner 1999).

Los trabajos de Monks (1981), Davis (1987), O’Connor (2000), Chaix y Méniel (2001) o Mateos Cachorro (2002) recopilan muchos de los métodos y técnicas utilizados para los análisis de estacionalidad. De entre todos ellos, Monks (1981) y Mateos Cachorro (2002) presentan una útil agrupación entre técnicas directas e indirectas.

Los métodos indirectos infieren la estacionalidad a través de datos como el sedimento y los procesos sedimentarios, los patrones de enterramiento y de movilidad o los estudios de densidad demográfica, más allá de las evidencias proporcionadas por la fauna y la flora. Según Monks (1981), no son los métodos más precisos, pero pueden ser técnicas alternativas para futuras investigaciones.

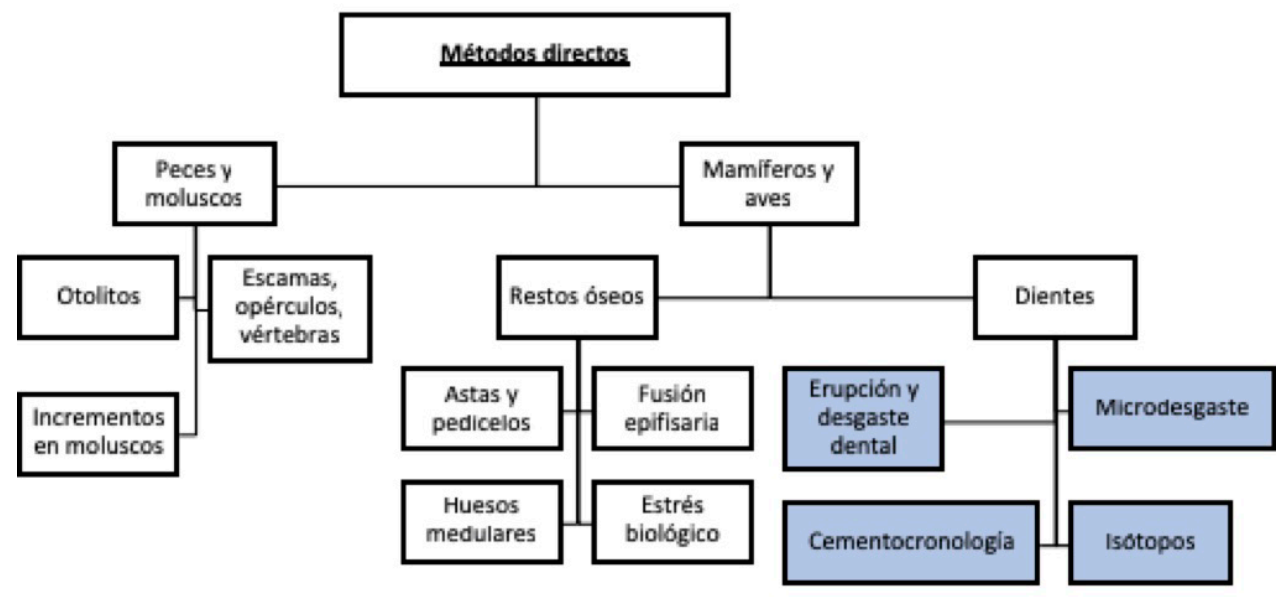

Figura 1. Esquema jerárquico de las subdivisiones de los métodos directos aplicados a los estudios de la estacionalidad. En azul, aquellas técnicas más susceptibles de complementarse entre sí.

Los métodos directos (Fig. 1) se basan en los restos faunísticos conservados en el yacimiento (Monks 1981; Mateos Cachorro 2002). Estos presentan tanto cambios fisiológicos como anillos de crecimiento en ciertas estructuras (llamados incrementos o annuli) que permiten establecer la edad del animal e inferir la estación de su muerte.

A través de este artículo se quiere profundizar sobre los métodos y técnicas aplicados a los análisis de estacionalidad, resaltando tanto sus virtudes como sus defectos, con el objetivo de verificar su funcionalidad y su grado de resolución, y desgranar así qué métodos pueden ser los más apropiados.

\section{Métodos y técnicas aplicadas a los estudios de estacionalidad}

Los trabajos de Monks (1981), Davis (1987), O’Connor (2000), Chaix y Méniel (2001) o 
Mateos Cachorro (2002) recopilan muchos de los métodos y técnicas utilizados para los análisis de estacionalidad. De entre todos ellos, Monks (1981) y Mateos Cachorro (2002) presentan una útil agrupación entre técnicas directas e indirectas.

\subsection{Métodos indirectos}

Los métodos indirectos infieren la estacionalidad a través de datos como el sedimento y los procesos sedimentarios, los patrones de enterramiento y de movilidad o los estudios de densidad demográfica, más allá de las evidencias proporcionadas por la fauna y la flora. Según Monks (1981), no son los métodos más precisos, pero pueden ser técnicas alternativas para futuras investigaciones.

\section{Granulometría de la matriz}

En ocasiones, el tamaño de las partículas de la matriz del sedimento depositado en los yacimientos atiende a variaciones estacionales. En los yacimientos costeros, la variación estacional en la dirección o velocidad del viento puede variar la cantidad de granos de arena de $0,08 \mathrm{~mm}$ presentes en la matriz. O que en yacimientos litorales o marinos sujetos a inundaciones estacionales, la proporción de granos de $0,2 \mathrm{~mm}$ o superior depende de la velocidad del agua (King 1971). Así, la granulometría de la matriz puede proporcionar información estacional siempre y cuando sea lo suficientemente abundante.

\section{Estudios químicos del suelo}

Determinados tipos de elementos dejan un rastro químico muy característico tras su descomposición. Su presencia en el sedimento permite inferir la recogida estacional de algunos recursos. Las altas concentraciones de mercurio encontradas en el estudio químico de la matriz del suelo del yacimiento de Neville, evidenciaron la recogida de especies de peces anádromos (Dincauze 1976).

\section{Coprolitos}

Los coprolitos son restos fósiles de heces que contienen residuos macro y microscópicos de fauna y flora, y pueden analizarse para conocer dicha dieta a través de los biomarcadores fecales (Bull et al. 2012; Sistiaga 2014a, 2014b).
Estos restos evidencian además las preferencias dietéticas y comportamiento subsistencial de las poblaciones de cazadores-recolectores, que pueden reflejar cambios estacionales. Sin embargo, es necesario tener en cuenta los recursos almacenados para un posterior consumo (Monks 1981).

\section{Evidencias Ecológicas}

En ocasiones, algunos comportamientos estacionales de animales, incompatibles con la presencia humana, informan del momento de ocupación de un yacimiento. La hibernación de los osos en los momentos rigurosos del año, las migraciones altitudinales de cabras, rebecos y otros ungulados según la estación, las migraciones estacionales de ñus entre el Serengueti y el Masái Mara, en el este de África. Lo mismo sucede con las fases de anidamiento de ciertas rapaces que, conscientes del riesgo que entraña convivir con humanos, tratan de buscar lugares con ausencia de presencia antrópica. En consecuencia, una acumulación de egagrópilas de ciertos búhos sería incompatible con la presencia humana.

\subsection{Métodos directos}

Los métodos directos se basan en datos obtenidos de los restos orgánicos de fauna conservados en el yacimiento. Se fundamentan tanto en los cambios fisiológicos como en las estructuras de incrementos, que permiten establecer la edad a partir de la adición sucesiva de anillos de crecimiento.

\subsubsection{Estudios de estacionalidad en peces y moluscos}

Los peces y los moluscos tienen un patrón de crecimiento continuo pero inconstante, condicionado por la abundancia de alimento y la temperatura (Davis 1987; Chaix y Méniel 2001). Si las condiciones no son favorables, el crecimiento se detiene, y viceversa. A pesar de algunos inconvenientes, Monks (1981), Davis (1987), Milner (1999), Chaix y Méniel (2001) y Mateos Cachorro (2002) acuerdan que son ejemplares válidos para los análisis de estacionalidad.

\section{Otolitos, vértebras, opérculos y escamas:}

En peces, se puede inferir la estacionalidad a partir de elementos que producen anillos de 
crecimiento, como los otolitos, opérculos y escamas y vértebras (ordenados según su relevancia). En especial, los otolitos son estructuras mineralizadas presentes en el oído interno de la mayor parte de los vertebrados, relacionados principalmente con el equilibrio (Davis 1987; Koch 2007). En peces óseos (teleósteos), los otolitos crecen diariamente creando incrementos que permiten inferir la estacionalidad. El estudio de los otolitos de los peces de Oronsay permitió correlacionar su tamaño con la edad del individuo (Mellars y Wilkinson 1980). Sabiendo la época de desove, se infiere la estacionalidad.

\section{Análisis de incrementos en moluscos}

La concha de los moluscos es una estructura cristalina de carbonato cálcico $\left(\mathrm{CaCO}_{3}\right)$ que se deposita durante la inmersión. Se producen dos tipos de líneas de crecimiento: las líneas externas (macrolíneas) y las líneas internas (microlíneas) (Monks 1981; Davis 1987; Milner 1999). Las macrolíneas son provocadas por cierto tipo de eventos, como el cese del crecimiento en invierno (Monks 1981; Milner 1999). En cambio, las microlíneas coinciden con el número de emersiones durante las mareas alta y baja.

El crecimiento puede llegar a detenerse en las estaciones frías, reiniciándose en las cálidas. Esto depende de variables como la latitud, la edad, las mareas, la variación de temperatura, etc. (Monks 1981).

Monks (1981), Davis (1987) y Milner (1999) recomiendan el uso de las microlíneas porque permiten el recuento de incrementos con láminas delgadas y de acetato. Además, pueden ser fotografiadas con rayos $\mathrm{X}$ y microscopios protónicos para diferenciar mejor los incrementos.

\subsubsection{Estudios de estacionalidad en mamí- feros $y$ aves}

\section{Astas y pedicelos de cérvidos}

Todos los machos de los géneros de la familia Cervidae poseen astas. Durante el celo otoñal, estos las usan en las luchas, mudándolas al final del invierno (desmogue), y volviéndoles a crecer en primavera, justo antes del celo. Entre los cérvidos, solo las hembras de Rangifer poseen astas, que mudan tras el nacimiento de las crías. Así, la aparición en el yacimiento de astas mudadas o aún fusionadas al cráneo podría indicar la estación (Monks 1981; Davis 1987; Milner 1999; Chaix y Méniel 2001).

Los pedicelos son dos protuberancias óseas sobre las que crecen las astas y presentan bandas que marcan las mudas sufridas. Durante el crecimiento del asta se crea una banda oscura en el pedicelo, alrededor del núcleo de crecimiento del asta. Y cuando esta ha madurado, se crea una banda clara. Esta sucesión de bandas oscuras y claras permite deducir la edad y estación de muerte del animal (Banfield 1960).

\section{Fusión epifisaria}

A medida que un individuo de una especie crece, el cartílago que une una articulación al cuerpo principal del hueso se osifica. Dependiendo del elemento anatómico y de la especie, la edad a la que se fusionan, calculada en meses a partir del mes de cría, es bien conocida, sobre todo para especies domésticas. La estacionalidad se obtiene de la suma de elementos fusionados y sin fusionar (Monks 1981; Davis 1987; O’Connor 2000; Chaix y Méniel 2001).

\section{Huesos medulares en aves}

Los "huesos medulares" se forman en las cavidades medulares de las hembras de ave como complemento de calcio y fósforo que necesita la cáscara de huevo en caso de déficit. Este fenómeno se produce durante el periodo de nidificación y es, a priori, un indicador de estacionalidad, pues en algunas especies se pueden identificar entre momentos de prenidificación y postnidificación (Rick 1975, 1979).

\section{Indicadores de estrés biológico}

Estos indicadores ponen de manifiesto momentos de cambios fisiológicos de un individuo. Entre ellos, la osteoporosis puede ser consecuencia de mudas de estructuras orgánicas y de estrategias reproductivas (Monks 1981). Las hipoplasias dentales o las líneas de Harris revelan insuficiencias nutricionales, siempre y cuando sean en momentos concretos. Y la grasa en ungulados varía según el estado reproductivo, la época del año, el sexo o la dieta (Mateos Cachorro 2002).

\subsection{Métodos directos basados en dientes}

Los dientes son uno de los elementos esqueléticos más duros. Su composición les permite 
soportar el deterioro provocado por el paso del tiempo, son buenos marcadores cronológicos, y no tienen ningún interés nutricional para los carnívoros (Payne 1973; Spinage 1973; Monks 1981; Grant 1982; Greenfield y Arnold 2008; Arceredillo y Díez 2009). Estas características hacen que sean los protagonistas de la mayoría de métodos de edad y estacionalidad más fiables y mejor conocidos.

Aun así, es necesario un buen entendimiento de la bioecología de las especies estudiadas (Milner 1999), una muestra representativa para paliar el efecto de la variabilidad intraespecífica y es aconsejable tener una colección de referencia en caso de duda (Davis 1987).

\subsubsection{Erupción y desgaste dental}

La edad de emergencia y sustitución de los dientes se conoce para muchas especies animales, en su mayoría especies domésticas de poblaciones actuales (Payne 1973, 1987; Deniz y Payne 1982; Grant 1982; Monks 1981; Davis 1987; O’Connor 2000; Chaix y Méniel 2001; Mateos Cachorro 2002; Steele 2002, 2004, 2005; Greenfield y Arnold 2008).

Para algunas especies salvajes, sobre todo taxones extintos, las secuencias de emergencia dental no siempre se conocen, debiéndose investigar o usar las secuencias de los taxones filogenéticamente más cercanos (Spinage 1971, 1972, 1973, 1976; Morrison y Whitridge 1997; Lubinski 2001; Arceredillo y Díez 2009).

Así, las piezas dentales pueden determinar la estación de muerte del individuo a partir de la edad (expresada en meses), calculando desde el mes de cría.

El desgaste dental determina la estacionalidad de dos maneras. Por un lado, estudiando los cambios morfológicos de la superficie oclusal de los dientes, cuyas etapas están asociadas a unas clases de edad más o menos definidas (Payne 1973, 1987; Deniz y Payne 1982; Grant 1982; Monks 1981; Davis 1987; O’Connor 2000; Chaix y Méniel 2001; Lubinski 2001; Greenfield y Arnold 2008).

Por otro, la altura de la corona permite determinar la edad de los individuos a través de una fórmula creada por Spinage (1971, 1972, 1976) y modificada por Klein (1981) y Klein y Cruz-Uribe (1983, 1984), y posteriormente por Steele (2002).
La erupción y el desgaste dental son dos métodos que tradicionalmente se han aplicado conjuntamente, desde el inicio de los estudios zooarqueológicos, por su sencilla aplicación sobre las muestras, tanto en laboratorio como en campo, sin necesidad de preparación previa (Spinage 1971, 1972; Monks 1981; Davies 1987; O’Connor 2000; Lubinski 2001; Steele 2002).

\subsubsection{Microdesgaste}

El microdesgaste estudia las adaptaciones dietéticas de taxones de herbívoros, tanto fósiles como actuales. Este método examina los rasgos microscópicos de la superficie oclusal del esmalte dental, producidos por el alimento consumido en un marco temporal muy reducido (Solounias y Semprebon 2002; Rivals y Deniaux 2005; Rivals y Solounias 2007; Rivals et al. 2007, 2009a, 2009b; Rivals y Semprebon 2012; Sánchez-Hernández et al. 2014; Rodríguez-Hidalgo et al. 2016).

Sin embargo, también se ha aplicado a taxones de primates (Semprebon et al. 2004), carnívoros (Goillot et al. 2009; Xafis et al. 2017) o humanos (DeSantis et al. 2013), en los que se han identificado caracteres similares, con resultados igualmente satisfactorios.

En general, estos rasgos se presentan en forma de fosas o arañazos y sus variantes: fosas pequeñas y grandes; arañazos finos, ásperos o cruzados, etc. Las diferentes combinaciones permiten clasificar a las especies entre ramoneadores estrictos, pascícolas/pastadoras estrictas o especies de alimentación mixta (Solounias y Semprebon 2002).

El método, aplicado en herbívoros desde los años 70, analizaba el microdesgaste con microscopio SEM a altos aumentos (500x) (Solounias y Semprebon 2002; Semprebon et al. 2004; Rivals y Deniaux 2005). Sin embargo, Solounias y Semprebon (2002) desarrollaron una variante más sencilla y rápida sobre los segundos molares, inferiores y superiores $\left(\mathrm{M}_{2} / \mathrm{M}^{2}\right)$, con un estereomicroscopio a bajos aumentos (35x), simplificando significativamente el proceso. Recientemente, Xafis et al. (2017) han mostrado que con el estudio del microdesgaste de los premolares se obtendrían resultados similares a los alcanzados a través del estudio de los molares. 
A

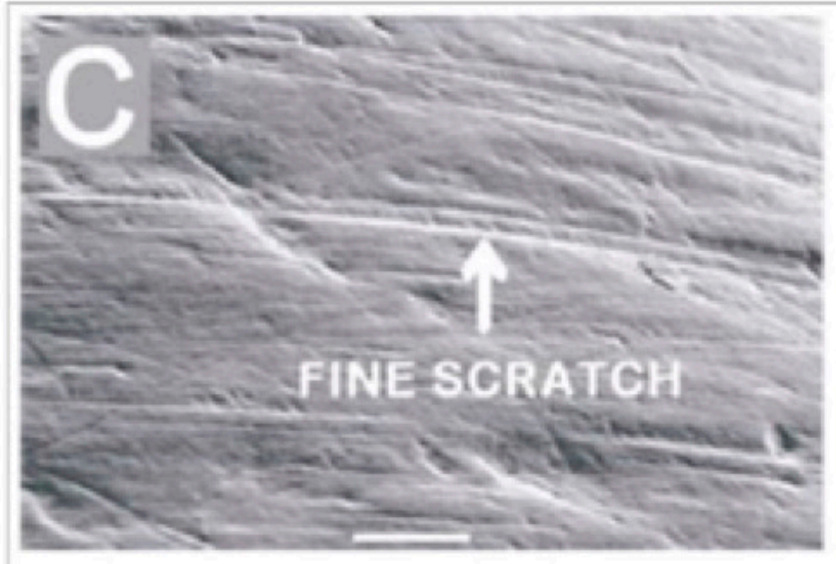

B

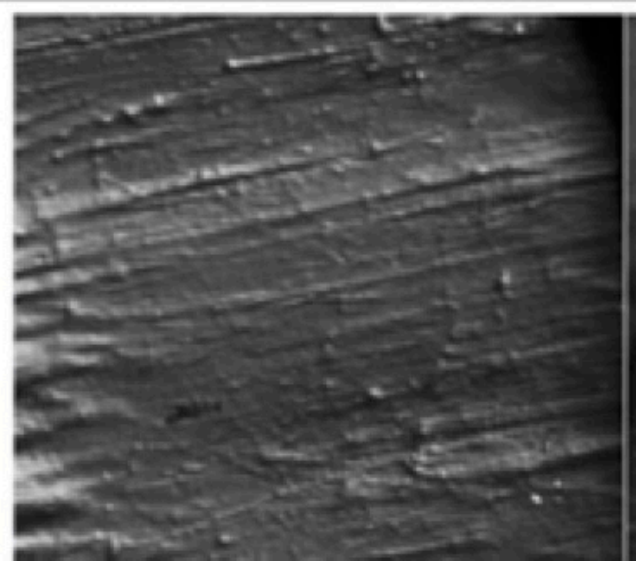

Figura 2. Imagen del esmalte dental en el que se observan las características que produce el microdesgaste: A) Fotografía a 500x bajo microscopio electrónico (Solounias y Semprebon 2002). B) Fotografía a 35x bajo estereomicroscopio (Florent).

Las clasificaciones dietéticas parten de las bases de datos creadas por Solounias y Semprebon (2002). Los herbívoros actuales se agrupan atendiendo al número de arañazos y fosas y los fósiles se comparan con aquellos cuyas dietas son conocidas. Genéricamente, las especies ramoneadoras tienen un bajo promedio del número de arañazos (0-17), y las pastadoras uno alto $(17,5-29,5)$, debido a los fitolitos y a las partículas de polvo y arena del alimento. El resto de rasgos ayudan a clasificar a las especies de alimentación mixta en categorías más precisas y matizan las tradicionales.

Las interpretaciones estacionales también se basan en el promedio de arañazos y fosas. $\mathrm{Si}$ los cambios estacionales determinan el tipo de vegetación (Rivals et al. 2009a, 2009b; Rodríguez-Hidalgo et al. 2016), la estación fría produciría un desplazamiento hacia hábitos dietéticos extremos: un aumento en el número de arañazos para las especies pastadoras y una disminución en las especies ramoneadoras, y viceversa.

Por último, el coeficiente de variación (CV) de la densidad de arañazos permite evaluar la duración de los asentamientos. Según estos autores, el CV refleja la variabilidad del comportamiento forrajeador de los animales: cuanto mayor sea el rango de alimentos de los que se sustenta el animal, mayor será la variación del microdesgaste, y viceversa. En concreto, un bajo valor del CV implicaría poca variabilidad, es decir, ocupaciones cortas; un alto valor del $\mathrm{CV}$ implicaría bastante variabilidad $\mathrm{y}$, o bien ocupaciones de larga duración, o bien una serie de ocupaciones cortas en diferentes momentos (Rivals et al. 2009a, 2009b; Rivals et al. 2015)

Para finalizar, es obligatorio citar la relación existente entre el microdesgaste y el mesodesgaste. El mesodesgaste es capaz de revelar el tipo de dieta adoptada por la fauna durante un marco temporal bastante largo, desvelando adaptaciones ecológicas a nichos inesperados para determinados tipos de especies, pero no tiene aplicación estacional. Desarrollada por Fortelius y Solounias (2000), el mesodesgaste estudia el grado de desgaste atricional (diente contra diente) y abrasivo (alimento contra diente) de la superficie oclusal del esmalte, que deja un relieve acusado y afilado y otro llano y romo, respectivamente (Fortelius y Solounias 2000; Rivals et al. 2007, 2009a, 2009b; Rivals y Semprebon 2012, Saarinen et al. 2015; Saarinen y Lister 2016).

\subsubsection{Cementocronología:}

El cemento es un tejido que forma parte de las raíces de los dientes, desde el cuello hasta el ápice de la raíz. Se genera durante toda la vida del animal y nunca se reabsorbe ni se remodela (Koch 2007; Britton et al. 2009; Rendu 2010; Fabre et al. 2011; Julien et al. 2012, 2015; Yravedra et al. 2016b). Este tejido está formado por tres tipos de componentes: hidroxiapatito en un $60-65 \%$, colágeno en un $20-25 \%$ y agua en un 10\% (Naji et al. 2015; vide contra, Lieberman 1994; Pérez Ripoll et al. 2001). 
Hay dos tipos de fibras de colágeno: las extrínsecas o fibras de Sharpey, que anclan el diente al ligamento periodontal en posición oclusal, y las intrínsecas (Lieberman 1994; Pérez Ripoll et al. 2001; Greenfield et al. 2015; Naji et al. 2015), que a su vez forman dos tipos de cemento: el acelular o primario y el celular o secundario.

El cemento acelular se compone en su mayoría por fibras de Sharpey, sintetizadas por el ligamento periodontal y mineralizadas por los cementoblastos de manera constante y lenta. El resultado es un tejido muy compacto y mineralizado (Lieberman 1994; Pike-Tay et al. 1999; Pérez Ripoll et al. 2001; Naji et al. 2015).

En cambio, el cemento celular, localizado en el extremo apical de la raíz, está compuesto por una mayor proporción de fibras de colágeno intrínsecas. Es más grueso que el acelular y su mineralización es más rápida e irregular (Lieberman 1994; Pérez Ripoll et al. 2001).
En conclusión, el cemento acelular es más útil para la estacionalidad por la regularidad y constancia con la que se deposita (Lieberman 1994; Pérez Ripoll et al. 2001; Greenfield et al. 2015; Naji et al. 2015).

Tras seccionar el diente para realizar láminas delgadas, y examinarlo bajo un microscopio de luz polarizada, el cemento acelular presenta un bandeado alternante de líneas translúcidas y opacas de diferentes tamaños. En estaciones frías, la cementogénesis se ralentiza, dando una banda más estrecha, mineralizada y opaca (annuli), mientras que en las estaciones cálidas es más rápida, generando una banda más ancha, menos mineralizada y translúcida. Conociendo la época de cría, la alternancia de bandas anchas y estrechas proporciona la edad, y el progreso de la última banda registrada, la estación (Lieberman et al. 1990; Lieberman 1994; Burke y Castanet 1995; Pike-Tay et al. 1999; Pérez Ripoll et al. 2001; Rendu 2010; Greenfield et al. 2015; Naji et al. 2015).

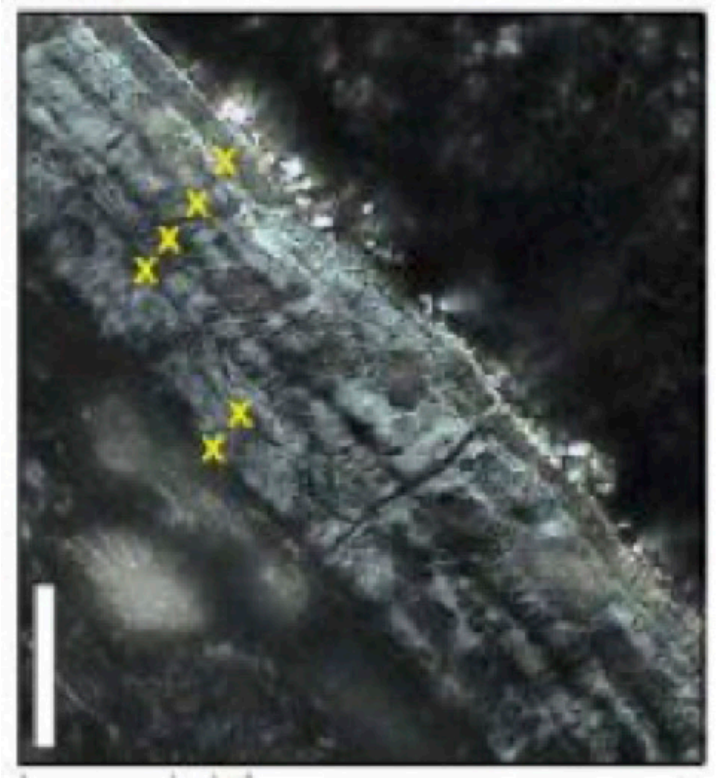

Figura 3. Imagen de las bandas oscuras y claras de cemento bajo luz polarizada (Naji et al. 2015).

Las causas de estas diferencias durante la cementogénesis podrían ser: 1) el estado nutricional, que da lugar a bandas hipo e hipermineralizadas; 2) los ciclos hormonales reproductivos, que afectan a las hormonas del crecimiento; y/o 3) las fuerzas biomecánicas durante la masticación, que orienta las fibras de Sharpey según la dureza de los alimentos (Lieberman et al. 1990; Lieberman 1994; Pike-Tay et al. 1999; Pérez Ripoll et al. 2001; Greenfield et al. 2015; Naji et al. 2015).

\subsubsection{Análisis de isótopos}

Los isótopos son átomos de un mismo elemento químico con idéntico número atómico (Z), pero diferente número másico (A) (Davis 1987). Se clasifican en isótopos estables, cuya vida media alcanza los 3.000 ma., e isótopos inestables o radiactivos, que emiten radiación durante su descomposición a un ritmo constante y conocido, y acaban convirtiéndose en otros elementos (Rozanski y Gonfiantini 
1990). Mientras que isótopos estables como el ${ }^{18} \mathrm{O},{ }^{13} \mathrm{C},{ }^{15} \mathrm{~N}$ y otros se han utilizado para reconstrucciones paleoclimáticas y paleoecológicas, los inestables se han usado en técnicas de datación: ${ }^{14} \mathrm{C},{ }^{40} \mathrm{~K} /{ }^{40} \mathrm{Ar}$ o las series de uranio-torio (Rozanski y Gonfiantini 1990; Koch 2007). Aunque hay una amplia variedad de isótopos (ib.), para los estudios paleoclimáticos, paleoecológicos y de estacionalidad, los más útiles son el $\delta^{18} \mathrm{O}, \delta^{13} \mathrm{C} y{ }^{87} \mathrm{Sr} /{ }^{86} \mathrm{Sr}$.

En vertebrados, estos isótopos se obtienen preferentemente del esmalte dental, compuesto en más de un $95 \%$ por mineral de bioapatita, o hidroxiapatita (Koch 2007), cuya fórmula química es: $\mathrm{Ca}_{10}\left[\mathrm{PO}_{4}\right]_{6}[\mathrm{OH}]_{2}$ (según Koch (2007) frente a Hoppe et al. (2004)). El valor de $\delta^{18} \mathrm{O}$ se extrae de los grupos de fosfatos $\left(\mathrm{PO}_{4}\right)^{3-}$ o de los grupos de carbonatos $\left(\mathrm{CO}_{3}\right)^{2-}$ que sustituyen a los grupos hidroxilo $(\mathrm{OH})^{-}$, citándose como $\delta^{18} \mathrm{O}_{\mathrm{p}}$ y $\delta^{18} \mathrm{O}_{\mathrm{c}}\left(\delta^{18} \mathrm{O}_{\mathrm{p}-\mathrm{c}}\right.$ en adelante) respectivamente. $\mathrm{El} \delta^{13} \mathrm{C}$ se extrae de los citados grupos de carbonatos, y los valores de ${ }^{87} \mathrm{Sr} /{ }^{86} \mathrm{Sr}$, del catión de estroncio ( $\mathrm{Sr}$ ) que sustituye al calcio (Ca) (Luz et al. 1984; Koch et al. 1989; Stuart-Williams and Schwarcz 1997; Frickle et al. 1998; Hoppe et al. 2004; Fisher y Fox 2007; Koch 2007; Britton et al. 2009; Fabre et al. 2011; Julien et al. 2012, 2015; Domingo et al. 2015; Yravedra et al. 2016b).

Estos isótopos se pueden obtener también de otras estructuras, como el colágeno óseo o la dentina, pero el esmalte dental es el tejido menos proclive a sufrir alteraciones diagenéticas (Fisher y Fox 2007; Koch 2007; Britton et al. 2009; Julien et al. 2012, 2015; Yravedra et al. 2016b). Aun así, en ocasiones el esmalte sufre alteraciones, sobre todo cuando el diente no está completamente formado y/o el esmalte no está mineralizado. En cambio, el proceso de mineralización del esmalte atenúa el valor de la señal isotópica del bioapatito, lo que podría interpretarse erróneamente como una menor variación de la temperatura local si no se corrige (Luz et al. 1984; Koch et al. 1989; Kohn 1996; Stuart-Williams and Schwarcz 1997; Frickle et al. 1998; Hoppe et al. 2004; Koch 2007; Britton et al. 2009; Fabre et al. 2011; Julien et al. 2012, 2015; Domingo et al. 2015; Yravedra et al. 2016b).

En invertebrados, el registro isotópico de la concha de los moluscos permite realizar reconstrucciones paleoambientales e inferir la estacionalidad. Las diferentes ratios de los isótopos de oxígeno $\left(\delta^{18} \mathrm{O} / \delta^{16} \mathrm{O}\right)$ y estroncio $\left({ }^{87} \mathrm{Sr} /{ }^{86} \mathrm{Sr}\right)$ presentes en el mar, y de las rela- ciones elementales de $\mathrm{Mg} / \mathrm{Ca}, \mathrm{Ba} / \mathrm{Ca}$ o $\mathrm{Sr} / \mathrm{Ca}$, están controladas por la temperatura de la superficie marina (SST). Y en última instancia, la SST está controlada por la temperatura ambiental. Así, por ejemplo, cuanto más rica en isótopos $\delta^{18} \mathrm{O}$ sea el agua del mar, menor será la SST, y viceversa (Davis 1987; Stecher et al. 1996; Andrus y Crowe 2000; Mannino et al. 2003, 2007; Takesue y Van Geen 2004; Carré et al. 2006; Sosdian et al. 2006; Wang et al. 2012; García-Escárzaga 2015).

\section{Isótopos en vertebrados: $\boldsymbol{\delta}^{18} \mathrm{O}$}

El principal isótopo utilizado en estudios paleoclimáticos, paleoecológicos y estacionales es $\delta^{18} \mathrm{O}$. Se explica porque:

1) el $\delta^{18} \mathrm{O}_{\mathrm{p} \text {-c }}$ del bioapatito dependen del valor isotópico del $\delta^{18} \mathrm{O}$ del agua corporal $\left(\delta^{18} \mathrm{O}_{\mathrm{ac}}\right)$ de los mamíferos terrestres, precipitando en equilibrio a temperatura corporal (Luz et al. 1984; Koch et al. 1989; Kohn 1996; Stuart-Wi1liams and Schwarcz 1997; Koch 2007; Britton et al. 2009; Fabre et al. 2011).

2) el $\delta^{18} \mathrm{O}_{\mathrm{ac}}$ refleja el $\delta^{18} \mathrm{O}$ de las aguas meteóricas locales $\left(\delta^{18} \mathrm{O}_{\mathrm{m}}\right)$, controladas por el ciclo global del $\delta^{18} \mathrm{O}$ (Paul et al. 1999) y condicionadas por las variables locales de aridez/humedad, temperatura y latitud (Luz et al. 1984; Koch et al. 1989; Kohn 1996; Stuart-Williams and Schwarcz 1997; Frickle et al. 1998; Hoppe et al. 2004; Koch 2007; Britton et al. 2009; Fabre et al. 2011; Julien et al. 2012, 2015; Domingo et al. 2015; Yravedra et al. 2016b).

3) Los valores más negativos del $\delta^{18} \mathrm{O}_{\mathrm{p}-\mathrm{c}}$ coinciden con las estaciones frías, y los valores más positivos con las estaciones más cálidas (ib.).

Hay factores fisiológicos que, por procesos de equilibrio metabólico, discriminan a favor de un isótopo de oxígeno sobre otro, enriqueciendo o empobreciendo el valor de $\delta^{18} \mathrm{O}$ (Luz et al. 1984; Koch et al. 1989; Kohn 1996; Koch 2007, Domingo et al. 2015). Entre ellos, los flujos de obtención y pérdida de oxígeno. Los primeros constan del vapor de agua (ca. $15 \%$ ) y el oxígeno inspirado (ca. $25 \%$ ), que sufren este fraccionamiento en los pulmones. Entre los de salida encontramos: la orina y las heces (ca. $40 \%$ ) y el $\mathrm{CO}_{2}$ expirado (ca. $25 \%$ ), la sudoración y evaporación transcutánea (ca. $35 \%$ ), que se fraccionan con respecto al agua corporal (Koch 2007).

Algunos factores medioambientales y ecológicos que controlan los valores de $\delta^{18} \mathrm{O}$ ya 
se han mencionado, como la aridez/humedad y la temperatura local. La evaporación provoca el enriquecimiento en ${ }^{18} \mathrm{O}$ en las aguas meteóricas y en las hojas de las plantas, cuyo índice aumenta con la evapotranspiración (Koch 2007; Domingo et al. 2015). Los grandes reservorios de agua, como lagos o pantanos, atenúan o eliminan las variaciones isotópicas estacionales por la homogeneización del agua en los acuíferos (Stuart-Williams and Schwarcz 1997; Hoppe et al. 2004). Asimismo, las migraciones estacionales de la fauna motivadas por la temperatura provocan una variabilidad $\delta^{18} \mathrm{O}_{\mathrm{p}-\mathrm{c}}$ reducida (Britton et al. 2009).

\section{Isótopos en vertebrados: $\boldsymbol{\delta}^{13} \mathrm{C}$}

La vegetación es la principal fuente de carbono para los herbívoros terrestres. El carbonato de la bioapatita se deriva principalmente del bicarbonato sanguíneo. El fraccionamiento en mamíferos que sucede a temperatura corporal produce un enriquecimiento en ${ }^{13} \mathrm{C}$ del 12 $14 \%$ para ungulados salvajes y un $c a$. $9 \%$ para los carnívoros, pues el $\delta^{13} \mathrm{C}$ aumenta con el nivel trófico (Koch 2007; Domingo et al. 2015; Hoppe et al. 2004; Julien et al. 2012, 2015; Domingo et al. 2015; Yravedra et al. 2016b).

Además, $\delta^{13} \mathrm{C}$ varía dependiendo de las vías fotosintéticas de las plantas: C3, C4 o CAM. La vía C3 se da en todos los árboles y mayoría de arbustos, hierbas y pastos en regiones templadas. Este tipo de plantas discriminan más que las $\mathrm{C} 4$ y CAM contra el ${ }^{13} \mathrm{C}$ durante la fotosíntesis. Afortunadamente, la amplia variación de $\delta^{13} \mathrm{C}$ (rango: $-35 \%$ a $-22 \%$; media: $-27 \%$ ) permite diferenciar nichos ecológicos entre plantas C3 por variaciones medioambientales y fisiológicas como la iluminación, la humedad, la temperatura o la evapotranspiración (Hoppe et al. 2004; Koch 2007; Domingo et al. 2015).

$\mathrm{La}$ vía $\mathrm{C} 4$ se dan en algunos juncos, dicotiledóneas y hierbas, e incrementan su presen- cia en regiones áridas y húmedas. En este caso los valores de $\delta^{13} \mathrm{C}$ son mayores (media: $c a$. $-13 \%$; rango: $-19 \%$ a $-9 \%$ ). Las plantas dicotiledóneas CAM están presentes también en regiones áridas, con valores similares a las $\mathrm{C} 4$, mientras que las CAM de ambientes húmedos tienen valores intermedios entre las C3 y las C4 (Hoppe et al. 2004; Koch 2007; Julien et al. 2012, 2015; Domingo et al. 2015; Yravedra et al. 2016b).

Así, la poca o mucha variabilidad de los valores isotópicos de $\delta^{13} \mathrm{C}$ permiten reconstruir la ecología y la dieta de los taxones analizados (Hoppe et al. 2004; Koch 2007; Julien et al. 2012, 2015; Domingo et al. 2015; Yravedra et al. 2016b).

\section{Estacionalidad mediante análisis de isóto- pos: los caballos de Schöningen}

Aunque otros trabajos de isótopos sobre dientes estudian la estación de muerte de diferentes animales (Koch et al. 1989; Stuart-Williams and Schwarcz 1997), y aunque Fisher y Fox (2007) ya los usaron para determinar el tipo de acumulación del yacimiento de Dent (Colorado, EE. UU.), el trabajo de Julien et al. (2015) muestra una nueva aplicación. En este trabajo se combina la variación intradental y la variabilidad interindividual de los valores del $\delta^{18} \mathrm{O}$ y del $\delta^{13} \mathrm{C}$ para investigar los patrones estacionales registrados a través de $\delta^{18} \mathrm{O}$, así como el origen múltiple o único de la acumulación del yacimiento de Schöningen.

Julien et al. (2015) toma dos tipos de muestras en dientes de caballos. Las primeras son longitudinales al eje de crecimiento del diente, que proporcionan valores medios de $\delta^{18} \mathrm{O}$. Las segundas son en serie, de pequeñas dimensiones, transversales al eje dental, desde la corona hasta el cérvix, con el objetivo de representar las variaciones estacionales del $\delta^{18} \mathrm{O}$ durante el crecimiento de dicho diente (Fabre et al. 2011; Julien et al. 2012, 2015; Yravedra et al. 2016b). 

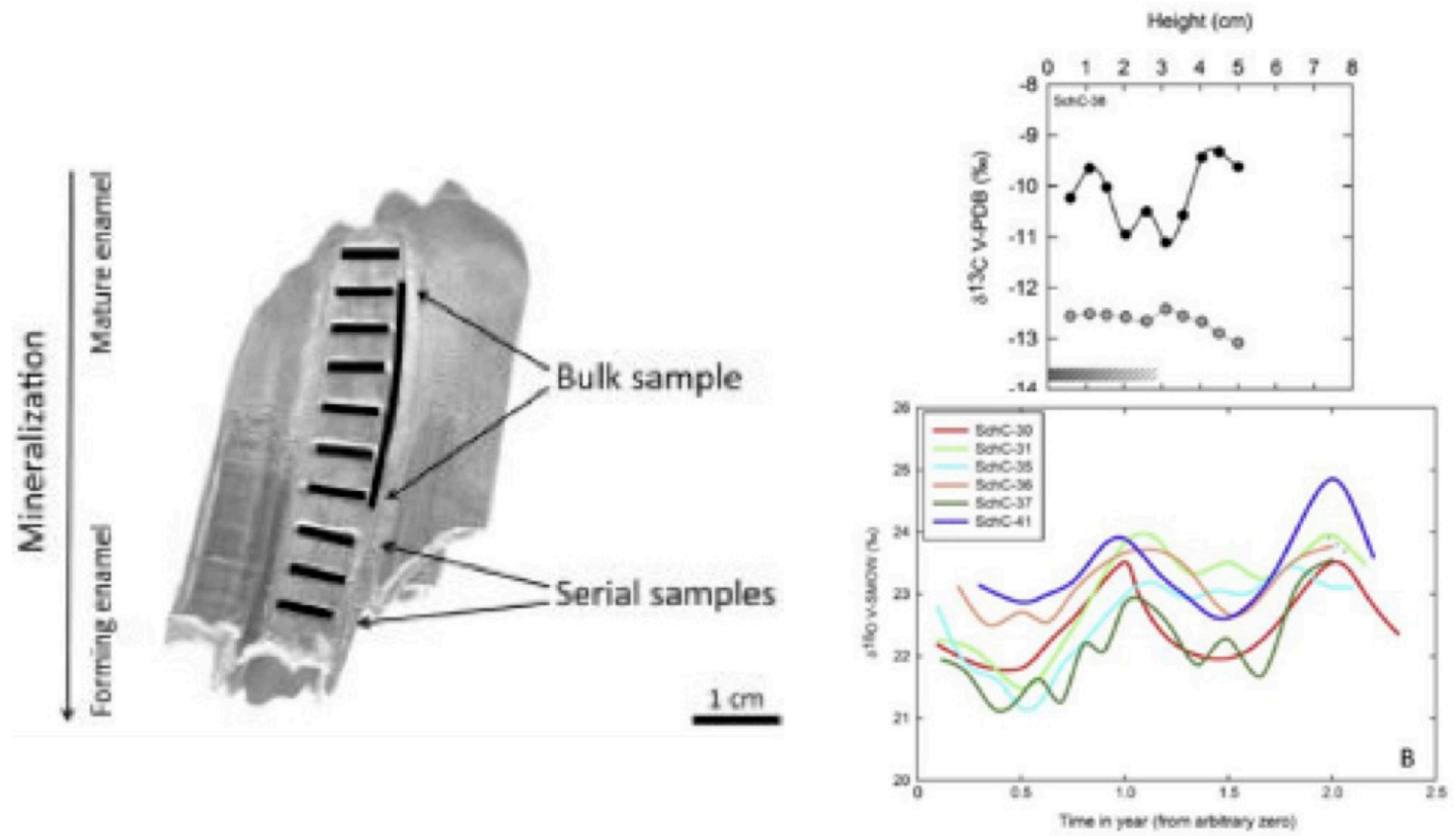

Figura 4. A) Imagen de los dos tipos de muestreo en dientes de caballos de Schöningen. B) Ejemplo de los resultados de $\delta 18 \mathrm{O}$ y $\delta 13 \mathrm{C}$ del muestreo en serie. C) Ejemplo de los valores de $\delta 18 \mathrm{O}$ y $\delta 13 \mathrm{C}$ de los muestreos longitudinales, que además ejemplifican el carácter múltiple de la acumulación de los caballos de Schöningen (Julien et al. 2015).

La conclusión a la que llegan Julien et al. (2015) es que los caballos de Schöningen pertenecen a diferentes acumulaciones debido a tres motivos:

1) La variabilidad de $\delta^{18} \mathrm{O}$ y $\delta^{13} \mathrm{C}$ de las muestras longitudinales concuerdan con el patrón registrado en dientes de caballos fósiles de diferentes yacimientos con múltiples niveles.

2) Los valores de las muestras en serie evidencian una mezcla de individuos emparentados y no emparentados.

3) Las muestras en serie revelan diferentes estaciones de muerte, registrada por la última señal del $\delta^{18} \mathrm{O}$ de los dientes inmaduros de caballos, evidenciando una acumulación múltiple.

Esta novedosa línea de investigación es muy interesante para los estudios de estacionalidad, ya que abre otra vía para esta área, sobre todo cuando se puede complementar con la técnica del microdesgaste (Rivals et al. 2014) o la cementocronología.

\section{Discusión}

Los estudios de estacionalidad han evolucionado con los años, apareciendo nuevas técnicas que, sin solucionar los problemas de los métodos tradicionales, al menos permitían obte- ner más datos. A su vez, esto ha permitido una aproximación heterogénea al comportamiento, a la gestión de los recursos y a la movilidad de los grupos humanos que ocuparon los yacimientos arqueológicos. El hecho de que no se consiga paliar los sesgos de dichas técnicas, propicia la oportunidad de complementar varios métodos, profundizando en el análisis de las poblaciones estudiadas. No obstante, es fundamental comprender a fondo la técnica empleada y conocer bien la bioecología de las especies de estudio, de manera que se aplique correcta (Milner 1999).

En este sentido, las técnicas citadas en este artículo como indirectas no son exactamente precisas, por lo que no permiten extraer conclusiones sobre el momento de ocupación de los yacimientos. Por ello, no son consideradas válidas para los estudios de estacionalidad, lo que no implica que no puedan ser expuestas para alentar futuros estudios estacionales través técnicas indirectas (Monks 1981).

A pesar de esto, en ocasiones pueden tenerse en cuenta las indicaciones de las técnicas indirectas al ofrecer aproximaciones relativas. Las acumulaciones de egagrópilas, por ejemplo, sugieren fases de anidamiento reiterado de rapaces en periodos distintos a las ocupaciones humanas. 
Las técnicas directas basan su metodología en los restos arqueológicos faunísticos, lo que les aporta una ventaja sustancial con respecto a las indirectas. Sin embargo, no todas son igualmente válidas, pues algunas muestran los mismos sesgos que las técnicas indirectas, su resolución impide inferir estacionalidad, como mucho, podrían permitir inferir la edad relativa de muerte del individuo, y no en todos los casos:

1) La estacionalidad mediante astas de cérvidos no es demasiado precisa. Los ejemplares más jóvenes y más viejos pueden no mudar hasta la primavera. Además, las astas pueden recogerse en cualquier momento del año, por ser muy apreciadas como materia prima para la fabricación de industrias óseas y arte mueble (Davis 1987; Milner 1999). Así, la resolución temporal del método abarca de seis a nueve meses de error.

2) Los pedicelos de cérvidos infieren la edad de muerte del individuo gracias al recuento de los annuli que presentan. Sin embargo, el tejido óseo interno limita la creación de bandas oscuras a cuatro anillos, acotando la edad a los primeros cinco años de vida. Además, la edad también provoca el desvanecimiento de las bandas. Así, la estacionalidad a partir de los pedicelos debe considerarse con absoluta precaución (Monks 1981).

3) En la fusión de las epífisis es fundamental comprender el papel que juega la variabilidad intraespecífica. Hay individuos y especies que maduran más rápido que otras, y otros factores, como la castración o el estado nutricional, afectan al rango de osificación (Monks 1981; Davis 1987; O’Connor 2000; Chaix y Méniel 2001). Pero el verdadero problema es la conservación de las epífisis, pues su baja densidad ósea y su rico contenido en grasas las hace propensas a desaparecer por la acción de agentes tafonómicos, como los carnívoros o la acidez del suelo (O’Connor 2000; Yravedra 2006)
4) Los huesos medulares de aves indican potencialmente la estacionalidad. Sin embargo, todavía no se conocen bien las especies de aves que crean estas estructuras, ni cómo su presencia en los huesos en periodos de prey postnidificación varían según la especie, el tipo de dieta y el estado nutricional y de salud del individuo. Además, el calcio puede ser obtenido por otras vías más allá de los huesos medulares (Rick 1975, 1979). La estacionalidad a través de esta técnica es, sencillamente, incierta (Monks 1981).

5) En peces, los otolitos, escamas, vértebras y opérculos también poseen estructuras de crecimiento que relacionan la edad con la estación de captura. Sin embargo, las escamas están sujetas a los daños externos. Opérculos y escamas pueden presentar anillos falsos. En vértebras, los incrementos aparecen con un retraso de mes y medio. Finalmente, los cambios producidos por causas somáticas y medioambientales desconocidas hacen de estas técnicas métodos fundamentalmente secundarios, más apropiados para calcular la edad que la estación de muerte (Le Cren 1947; Casteel 1976; Monks 1981).

6) Finalmente, las causas que provocan el estrés son complicadas de definir por la cantidad de variables implicadas que pueden afectar a los huesos, como el sedimento, la porosidad ósea o insuficiencias nutricionales prolongadas. Cuando el periodo de tiempo es indeterminado, es realmente complicado inferir la época de muerte (Monks 1981; Mateos Cachorro 2002). Por lo tanto, esta técnica también es inviable como método estacional.

El resto de técnicas que vienen a continuación, a pesar de tener desventajas y límites no resueltos, se consideran por los autores como métodos principales (Tabla 1). Aun así, algunos de estos métodos no son lo suficiente robustos como para realizar estudios de estacionalidad independientemente, por lo que sería preferible que se complementasen unos con otros para superar sus respectivas limitaciones. 


\begin{tabular}{|c|c|c|c|c|}
\hline Técnica & Tipo & Carácter & Ventajas & Limitaciones \\
\hline $\begin{array}{l}\text { Incrementos en } \\
\text { moluscos }\end{array}$ & Directa & $\begin{array}{l}\text { Método } \\
\text { principal }\end{array}$ & Resolución & $\begin{array}{l}\text { Variabilidad intraespecífica } \\
\text { Equipamiento } \\
\text { Subjetividad }\end{array}$ \\
\hline $\begin{array}{c}\text { Erupción y } \\
\text { desgaste dental }\end{array}$ & Directa & $\begin{array}{l}\text { Método } \\
\text { principal }\end{array}$ & $\begin{array}{l}\text { Económico/barato } \\
\text { Sencillo } \\
\text { Inmediato } \\
\text { Rápido } \\
\text { Sin preparación } \\
\text { No destructivo }\end{array}$ & $\begin{array}{l}\text { Variabilidad intraespecífica } \\
\text { Sólo infantiles } \\
\text { Muestra amplia } \\
\text { Cohortes amplias } \\
\text { Poco resolutivo }\end{array}$ \\
\hline Microdesgaste & Directa & $\begin{array}{l}\text { Método } \\
\text { principal }\end{array}$ & $\begin{array}{c}\text { Económico/barato } \\
\text { Rápido } \\
\text { Sencillo } \\
\text { Sin preparación } \\
\text { No destructivo } \\
\text { Interpretaciones paleoecológicas } \\
\text { y paleoambientales }\end{array}$ & Poco resolutivo \\
\hline Cementocronología & Directa & $\begin{array}{l}\text { Método } \\
\text { principal }\end{array}$ & $\begin{array}{l}\text { Resolutivo } \\
\text { Sin límite de edad ni sexo } \\
\text { Programas informáticos }\end{array}$ & $\begin{array}{c}\text { Preparación de las muestras } \\
\text { Equipamiento } \\
\text { Destructivo } \\
\text { Problemas biológicos (compactación del } \\
\text { cemento, falsos incrementos, incrementos } \\
\text { sin annuli). } \\
\text { Problemas tafonómicos (diagénesis, alte- } \\
\text { raciones químicas, weathering). } \\
\text { Experiencia }\end{array}$ \\
\hline Análisis de isótopos & Directa & $\begin{array}{l}\text { Método } \\
\text { principal }\end{array}$ & $\begin{array}{l}\text { Resolutivo. } \\
\text { Tipo de acumulación (múltiple } \\
\text { o única). } \\
\text { Interpretaciones paleoecológicas } \\
\text { y paleoambientales. }\end{array}$ & $\begin{array}{l}\text { Preparación de las muestras } \\
\text { Equipamiento } \\
\text { Destructivo } \\
\text { Factores atenuantes de la señal isotópica }\end{array}$ \\
\hline
\end{tabular}

Tabla 1. Comparación de los diferentes métodos directos principales, mostrando ventajas y limitaciones de cada uno de ellos.

El estudio de los incrementos internos en las conchas de los moluscos es una técnica válida para el estudio de la estacionalidad, que posee una notable resolución (Monks 1981; Davis 1987; Milner 1999), siempre y cuando se tengan en cuenta dos cuestiones. Primero, hay variables externas a los moluscos que afectan al crecimiento de la concha y pueden provocar su detención, como la temperatura, ataques de depredadores, la luz solar y lunar, etc. $(i b$.). Segundo, es necesario conocer muy bien la biología de las especies estudiadas al contar los incrementos, ya que el reinicio del crecimiento en primavera está sujeto a una gran variabilidad inter e intraespecífica que puede comprometer la integridad de los análisis $(i b$.$) .$

La erupción y desgaste dental han sido las técnicas más usadas en los estudios zooarqueológicos por su antigüedad y sencillez, lo cual permite aplicar la técnica sin preparación previa, incluso en campo (Spinage 1971, 1972; Monks 1981; Davies 1987; O’Connor 2000; Lubinski 2001; Steele 2002). Sin embargo, otras desventajas son inevitables.

Primero, la erupción solo se puede aplicar a mandíbulas y maxilares de individuos infantiles, con la excepción de los elefantes (Spinage 1973), y a priori, los dientes aislados tampoco pueden ser estudiados (Monks 1981). Otros factores, como la variabilidad intraespecífica en la erupción dental (Spinage 1973), el desajuste en la emergencia de los dientes inferiores y los superiores (Talbot y Talbot 1963), la domesticación (Monks 1981), pueden condicionar la secuencia de erupción dental y de desgaste de la especie en cuestión

En segundo lugar, el desgaste posee una amplia variabilidad una vez alcanzada la madurez. Los comportamientos nutricionales 
individuales, la dureza del alimento, la cantidad de partículas erosivas que este contiene, y que cambia con la latitud (Chaix y Méniel 2001), o el desfase entre erupción y desgaste en molares de bóvidos (Monks 1981; Davis 1987; O’Connor 2000; Chaix y Méniel 2001), pueden variar el patrón de desgaste (Spinage 1973; Monks 1981; O'Connor 2000). Aunque estos factores no inhabilitan la técnica, obligan a incluir cohortes con rangos de edad más amplios a medida que envejecen, lo que acaba restando resolución al método (Spinage 1971, 1973; Payne 1973; Deniz y Payne 1982; Grant 1982; Lubinski 2001; Greenfield y Arnold 2008; Arceredillo y Díez 2009).

Y en tercer lugar, se debe contar con la subjetividad de la técnica. El estudio se realiza a simple vista y la percepción del grado de desgaste puede variar entre investigadores. Esto conllevaría diferencias en la estimación de la edad, sobre o subestimando dicha edad y, por tanto, la adscripción errónea del individuo estudiado a la cohorte correspondiente (Spinage 1972, 1973; Payne 1973; Klein 1981; Klein y Cruz-Uribe 1983, 1984; Payne 1987; Steele 2002, 2004, 2005; Arceredillo y Díez 2009).

Aunque la medición de la altura de la corona puede paliar parcialmente la subjetividad del investigador, no corregirá la influencia de otras variables que influyan en el desgaste (Spinage 1972, 1973). De hecho, realmente esta técnica no permite inferir la estacionalidad, puesto que su aplicación obliga a la creación de cohortes que corresponden al $10 \%$ de la esperanza vida del individuo. En este sentido, Steele (2002) especifica que cuando la edad de un individuo no se clasifica correctamente en la cohorte debida, lo hace en una de las adyacentes, lo que genera un sesgo importante a la hora de calcular la estación de muerte. Aunque para Steele esto sea una prueba de la validez del método, es inviable determinar la estación de muerte a partir de la altura de la corona.

Por todo ello, esta técnica necesita de una muestra amplia que disminuya el efecto de todas estas variables. En ocasiones, esto representa un problema, pues no todos los yacimientos cuentan con suficientes especímenes. Además, inevitablemente los rangos de edad de las cohortes en los que se clasifican deben ser amplios, lo que limita la precisión y la resolución de la técnica (Monks 1981; Davis 1987; O’Connor 2000; Chaix y Méniel 2001). Por tanto, la erupción y desgaste dental y la altura de la corona son técnicas útiles para de- terminar los perfiles de edad de los conjuntos arqueológicos, pero no son válidas para definir con certeza la estacionalidad de los yacimientos. Esto hace que en los próximos años, su uso como técnica para determinar la estacionalidad se vea sustituida con mayor probabilidad por el microdesgaste, y por isótopos y/o cementocronología en menor medida. Aun así, esta técnica podría reducir sus problemas si se complementase con algunas de las técnicas más robustas, como isótopos o cementocronología.

El método de microdesgaste a bajos aumentos desarrollado por Solounias y Semprebon (2002) y Semprebon et al. (2004) facilita el estudio de grandes muestras en poco tiempo, de manera sencilla y sin necesitar muestras amplias, mejorando significativamente el proceso mediante microscopio SEM (Solounias y Semprebon 2002; Semprebon et al. 2004; Rivals y Deniaux 2005; Rivals et al. 2007). Solounias y Semprebon (2002) proporcionaron también una amplia base de datos comparativa de herbívoros, junto con algunos taxones extintos de équidos, a la que se han añadido más taxones en posteriores estudios (Semprebon et al. 2004; Goillot et al. 2009; Xafis et al. 2017) Además, permite el estudio de la duración de las ocupaciones humanas y de las adaptaciones ecológicas de la fauna (Rivals et al. 2009b; Rivals et al. 2015). Sin embargo, este método sigue dando una resolución temporal demasiado amplia de 3-6 meses, y en algunos casos y contextos, la estacionalidad sigue sin poder definirse. Los análisis de isótopos serían un buen complemento a esta técnica para paliar algunos de sus inconvenientes (Rivals et al. 2014), así como la cementocronología.

Semprebon et al. (2004) y Rivals et al. (2007) añaden que este método, robusto y replicable, no está sujeto a la subjetividad del investigador. Pero DeSantis et al. (2013) concluyen precisamente que las metodologías empleadas para analizar el microdesgaste son demasiado variables, y los resultados obtenidos, incomparables. Ya sea mediante SEM o a bajos aumentos $(35 \mathrm{x})$, la cuantificación de los rasgos del microdesgaste genera grandes sesgos, especialmente entre observadores con diferente grado de experiencia. Y esta inconsistencia pone en cuestión la reproducibilidad y comparabilidad del microdesgaste en dos dimensiones (es decir, SEM o bajos aumentos). Por el contrario, en tres dimensiones (3D), o DMTA (Dental Microwear Texture Analysis), se elimina el sesgo del observador y se dis- 
tinguen nichos ecológicos más sutiles y precisos más allá de las grandes categorías. Para ello emplea rasgos relativos a la profundidad, como la anisotropía, la escala de máxima complejidad, el volumen de relleno de textura, etc. (DeSantis et al. 2013). Sin embargo, las ventajas de esta técnica superan sus inconvenientes, lo que provocará un aumento del uso de esta técnica en detrimento del desgaste como indicador de la estación, como actualmente ya está ocurriendo.

La cementocronología es, posiblemente, una de las técnicas de estacionalidad más complejas (Mateos Cachorro 2002; Arceredillo y Díez 2009; Rendu 2010; Greenfield et al. 2015; Naji et al. 2015), pues entraña ciertas dificultades que se añaden al coste y al carácter destructivo de la técnica, y que son necesarias conocer previo a su uso (Rendu 2010; Naji et al. 2015).

Por un lado, algunos condicionantes biológicos pueden dar lugar a la compactación de las bandas de cemento o a la formación de falsos incrementos, sub o sobreestimando la edad, respectivamente. Excepcionalmente, pueden aparecer depósitos de cemento sin annulus como consecuencia de un intenso estrés. Y finalmente, la variabilidad intraespecífica que se puede dar entre individuos de una misma población de manera natural (Monks 1981; Lieberman et al. 1990; Rendu 2010; y Naji et al. 2015).

Por otro lado, existen factores de carácter tafonómico que provocan la remodelación del cemento durante su mineralización. La exposición subaérea o meteorización (weathering) puede provocar grietas en la unión entre la dentina y el cemento que conlleven el desprendimiento definitivo de éste, las alteraciones químicas producidas durante la digestión y/o regurgitación imposibilitan el recuento y la precipitación de minerales, su sustitución y recristalización durante la diagénesis puede modificar las capas de cemento, provocando estimaciones erróneas $(i b$.).

En cuanto a la interpretación, la estimación de la estacionalidad se hace midiendo el grosor de la última capa de cemento, utilizando las otras capas como referencia, permitiendo una mayor precisión (inicio, mitad o fin de la estación cálida, etc.) (Naji et al. 2015). Por tanto, esta técnica ofrece una mejor resolución que el resto de los métodos, con la excepción de los análisis de isótopos.

Por todo ello, Naji et al. (2015) apuntan la necesidad de adquirir un mínimo de experien- cia práctica en la interpretación para ser capaz de evitar estos problemas. Para ello, se puede recurrir a pruebas ciegas y a varios observadores para paliar parcialmente la subjetividad de su estudio. Adicionalmente, se pueden usar programas informáticos que ayuden a mejorar la calidad de las imágenes y a medir las dimensiones de las capas. Desgraciadamente, aún no se ha conseguido desarrollar satisfactoriamente un software de recuento automático de las capas de cemento (Naji et al. 2015). Sin embargo, estos autores destacan factores positivos del método, como la alta reproducibilidad del método en ciertas especies, y la potencialidad de este método en los estudios arqueológicos (ib.). Desde luego, si se consigue superar el carácter destructivo del método, esta técnica tiene muy buenas perspectivas de futuro, además de ser un complemento ideal para el resto de las tres técnicas principales, como también lo es el análisis de isótopos estables.

La aplicación de análisis de isótopos estables a la estacionalidad de yacimientos arqueológicos no ha sido una de las técnicas más comunes. El estudio de Julien et al. (2015) presenta una perspectiva novedosa porque han conseguido establecer la estacionalidad y el carácter múltiple o único de la acumulación a través de estos análisis, que se habían centrado eminentemente en reconstrucciones paleoambientales, ya fuesen sobre restos de moluscos o de mamíferos.

No obstante, su carácter semidestructivo, el coste y su complejidad limitan la aplicación de esta técnica. Y además, hay que añadir ciertos fenómenos ya citados que atenúan la señal isotópica registrada. Por el contrario, su resolución temporal es mayor que la del microdesgaste y similar a la de la cementocronología, compartiendo con esta la ventaja añadida de poder determinar tanto la estación como el estado de avance de ésta (Fisher y Fox 2007; Julien et al. 2015). Y además, también es posible determinar la duración de las ocupaciones humanas y la pertenencia a un o no a un mismo grupo (Julien et al. 2015). Es una mejora sustancial con respecto a aquellos métodos que solo precisan en términos de invierno vs. verano y similar, pero serán necesarias todavía más investigaciones que arrojen mejores resultados a esta prometedora técnica, además de ser un complemento ideal para la técnica del microdesgaste y/o cementocronología, siempre y cuando sea posible su aplicación (Rivals et al. 2014). 


\section{Conclusiones}

Ante la falta de trabajos que recogiesen los nuevos métodos que han surgido en los últimos tiempos sobre estudios de estacionalidad, el propósito de este artículo era recopilar las diferentes técnicas que se han desarrollado sobre ellos hasta la fecha. Sobre todo, se han recogido aquellos trabajos basados en restos óseos de fauna, explicando su metodología y destacando tanto sus virtudes como sus carencias.

En general, se podría decir que el principal inconveniente de los métodos de estacionalidad la variabilidad, inter o intraespecífica, la cual además afecta al grado de resolución y precisión de cada método, fundamental a la hora de determinar la viabilidad de un método estacional. Aunque la variabilidad afecta a cada método de manera diferente, siendo más o menos determinante según la técnica de que se trate, esto implica la obligación de conocer a fondo la eco-biología de las especies sobre las que se fundamentan los estudios con el fin de minimizar al máximo dicha variabilidad, para lo cual es aconsejable - dependiendo de la técnica - tanto una colección de referencia como una muestra.

De todos los métodos expuestos, este trabajo presenta una clara exposición de los cuatro que más se han aplicado hasta la fecha y/o que tienen mayores perspectivas de futuro: el desgaste dental, el microdesgaste, la cementocronología y el análisis de isótopos estables.

Se puede concluir que, de todos ellos, el menos propicio para estudiar la estacionalidad es el desgaste. Tanto la observación de los cambios sufridos en la superficie oclusal como la medición de la altura de la corona pueden servir para establecer una edad relativa de los individuos que componen el conjunto óseo. Pero en cuanto a la estacionalidad, es un método que tiene un rango de error muy amplio y una baja resolución, solo es válido en mandíbulas y maxilares de individuos infantiles y juveniles, de especies con un patrón de desgaste conocido. Además de la variabilidad intraespecífica ya mencionada, destacar también la subjetividad del método, aspecto que la medición de la corona palía, aunque no resuelve el resto de problemas mencionadas.

El microdesgaste palía muchos de los problemas de la mayoría de técnicas: es barata y fácil de aplicar, no es destructiva, no está suje- ta a la subjetividad del investigador y permite establecer tanto la duración de las ocupaciones como la estacionalidad, en el caso de que esta se pueda definir. Por el contrario, el grado de resolución no es demasiado alto, precisando normalmente entre época cálida o fría.

Los análisis de isótopos de oxígeno tienen mejores perspectivas de futuro, pues además de utilizarse para el estudio del paleoambiente, permite inferir la estacionalidad con una resolución algo mayor que el microdesgaste, y similar a la cementocronología, y permite también el carácter múltiple o único de la acumulación. Por todo ello, podría decirse que esta técnica complementa perfectamente al microdesgaste. Sin embargo, el carácter semidestructivo de la técnica, junto con la complejidad para llevarla a cabo en términos económicos y de equipamiento, son sus mayores inconvenientes, y también los mayores retos a batir en los próximos años.

Por último, la cementocronología es también otra de las técnicas que mejores resultados podría llegar a proporcionar, sobre todo si en un futuro se es capaz de aplicar un software de lectura que permita inferir la estación a través del grado de progreso de la última banda en formación. Al igual que los isótopos, esta es una técnica ideal para complementarla con los análisis del microdesgaste, o incluso con el propio análisis de isotopos estables. Aun así, su carácter destructivo es la mayor desventaja con la que cuenta este método.

Por todo ello, el campo de la estacionalidad es un ámbito fascinante que está abierto a un amplio abanico de oportunidades, y la combinación de los métodos de microdesgaste, cementocronología y análisis de isótopos estables puede arrojar resultados muy interesantes en los próximos años.

\section{Agradecimientos}

Gracias a la Dra. Alicia Marín Roldán por sus comentarios y aclaraciones. Gracias también al programa de becas de La Fundación La Caixa, sin cuya financiación este trabajo no hubiera sido posible. Se agradece al DST-NRF Centre of Excellence in Paleosciences (CoEPal) su colaboración en esta investigación. Las opiniones y conclusiones a las que han llegado los autores responsables del artículo no deben ser necesariamente atribuidas al CoE-Pal. 


\section{Bibliografía}

Arceredillo, D.; Díez, C. (2009): Age of death and seasonality based on ungulate tooth remains from the Upper Pleistocene site of Valdegoba (Burgos, Spain). Journal of Taphonomy 7: 75-91.

Banfield, A. W. F. 1960: The use of caribou antler pedicles for age determination. Journal of Wildlife Management, 24: 99-102.

Bargalló, A.; Gabucio, M.J.; Rivals, F. (2016): Puzzling out a palimpsest: Testing an interdisciplinary study in level O of Abric Romaní. Quaternary International 417: 51-65. https://doi.org/10.1016/j. quaint.2015.09.066

Britton, K.; Grimes, V.; Dau, J.; Richards, M.P. (2009): Reconstructing faunal migrations using intra-tooth sampling and strontium and oxygen isotope analyses: a case study of modern caribou (Rangifer tarandus granti). Journal of Archaeological Science 36: 1163-1172. doi:10.1016/j.jas.2009.01.003

Bull, I.D.; Lockheart, M.J.; Elhmmali, M.M.; Roberts, D.J.; Evershed, R.P. (2012): The origin of faeces by means of biomarker detection. Environtal International 27: 647-654.

Burke, A.; Castanet, J. (1995): Histological observations of cementum growth in horse teeth and their application to archaeology. Journal of Archaeological Science 22: 479-493.

Carrancho, Á.; Villalaín, J.J.; Vallverdú, J.; Carbonell, E. (2016): Is it possible to identify temporal differences among combustion features in Middle Palaeolithic palimpsests? The archaeomagnetic evidence: A case study from level O at the Abric Romaní rock-shelter (Capellades, Spain). Quaternary International 417: 39-50. https://doi.org/10.1016/j.quaint.2015.12.083

Carré, M.; Bentaleb, I.; Bruguier, O.; Ordinola, E.; Barrett, N.T.; Fontugne, M. (2006): Calcification rate influence on trace element concentrations in aragonitic bivalve shells: Evidences and mechanisms. Geochimica et Cosmochimica Acta 70: 4906-4920. doi:10.1016/j.gca.2006.07.019

Casteel, R.W. (1976): Fish remains in archaeology and paleoenvironmental studies. New York: Academic Press.

Chaix, L.; Méniel, P. (2001): Archéozoologie: les animaux et l'archéologie. Editions Errance, Paris.

Davis, S.J.M. (1987): The archaeology of animals. Batsford Ltd, London.

Deniz, E.; Payne, S. (1982): Eruption and wear in the mandibular dentition as a guide to ageing Turkish Angora goats. En Wilson, B.; Grigson, C.; Payne, S. (ed.): Ageing and Sexing Animal Bones from Archaeological Sites. British Archaeological Reports, British Series, vol. 109. BAR, Oxford: 153-206.

DeSantis, L.R.G.; Scott, J.R.; Schubert, B.W.; Donohue, S.L.; McCray, B.M.; Van Stolk, C.A.; Winburn, A.A.; Greshko, M.A.; O'Hara, M.C. (2013): Direct Comparisons of 2D and 3D Dental Microwear Proxies in Extant Herbivorous and Carnivorous Mammals. PLOS ONE 8: e71428. https://doi. org/10.1371/journal.pone.0071428

Dincauze, D. F. (1976): The Neville Site: 8,000 years at Amoskeag, Manchester, New Hampshire. Peabody Museum Monographs, 4.

Domingo, L.; Pérez-Dios, P.; Hernández Fernández, M.; Martín-Chivelet, J.; Ortiz, J.E.; Torres, T. (2015): Late Quaternary climatic and environmental conditions of northern Spain: An isotopic approach based on the mammalian record from La Paloma cave. Palaeogeography, Palaeoclimatology, Palaeoecology 440: 417-430. doi:10.1016/j.palaeo.2015.09.017

Fabre, M.; Lécuyer, C.; Brugal, J.-P.; Amiot, R.; Fourel, F.; Martineau, F. (2011): Late Pleistocene climatic change in the French Jura (Gigny) recorded in the d180 of phosphate from ungulate tooth enamel. Quaternary Research 75: 605-613.

Fisher, D.C.; Fox, D.L. (2007): Season of death of the Dent mammoths. En Brunswig, R.H.; Pitblado, B.L. (ed.): Paleoindian Archaeology: From the Dent Site to the Rocky Mountains. The University Press of Colorado, Boulder, Colorado: 123-153.

Fortelius, M.; Solounias, N. (2000): Functional characterization of ungulate molars using the abrasion-attrition wear gradient: a new method for reconstructing paleodiets. American Museum Novitates 1-36.

Frickle, H.C.; Clyde, W.C.; O’Neil, J.R. (1998): Intra-tooth variations in $\delta 18$ O (PO 4) of mammalian tooth enamel as a record of seasonal variations in continental climate variables. Geochimica et Cosmochimica Acta 62: 1839-1850.

García-Escárzaga, A.; Moncayo, S.; Gutiérrez Zugasti, I.; González Morales, M. R.; Martín- Chivelet, J.; Cáceres, J. O. (2015): Mg/Ca ratios measured by laser induced breakdown spectroscopy (LIBS): a new approach to decipher environmental conditions. Journal of Analytical Atomic Spectrometry 30: 1913-1919. DOI: 10.1039/C5JA00168D 
Goillot, C.; Blondel, C.; Peigné, S. (2009): Relationships between dental microwear and diet in Carnivora (Mammalia) - Implications for the reconstruction of the diet of extinct taxa. Palaeogeography, Palaeoclimatology, Palaeoecology 271: 13-23. doi:10.1016/j.palaeo.2008.09.004

Grant, A. (1982): The use of tooth wear as a guide to the age of domestic ungulates. En Wilson, R.; Grigson, C.; Payne, S. (ed.): Ageing and Sexing Animal Bones from Archaeological Sites. British Archaeological Reports, British Series, vol. 109. BAR, Oxford: 91-108.

Greenfield, H.J.; Arnold, E.R. (2008): Absolute age and tooth eruption and wear sequences in sheep and goat: determining age-at-death in zooarchaeology using a modern control sample. Journal of Archaeological Science 35: 836-849. doi:10.1016/j.jas.2007.06.003

Greenfield, H.J.; Moore, N.C.; Steppan, K. (2015): Estimating the age- and season-of-death for wild equids: a comparison of techniques utilising a sample from the late neolithic site of Bad Buchau-Dullenried, Germany. Open Quaternary 1. doi:10.5334/oq.ac

Hoppe, K.A.; Amundson, R.; Vavra, M.; McClaran, M.P.; Anderson, D.L. (2004): Isotopic analysis of tooth enamel carbonate from modern North American feral horses: implications for paleoenvironmental reconstructions. Palaeogeography, Palaeoclimatology, Palaeoecology 203: 299-311. doi:10.1016/ S0031-0182(03)00688-6

Julien, M.-A.; Bocherens, H.; Burke, A.; Drucker, D.G.; Patou-Mathis, M.; Krotova, O.; Péan, S. (2012): Were European steppe bison migratory? 18O, 13C and $\mathrm{Sr}$ intra-tooth isotopic variations applied to a palaeoethological reconstruction. Quaternary International 271: 106-119. doi:10.1016/j.quaint.2012.06.011

Julien, M.-A.; Rivals, F.; Serangeli, J.; Bocherens, H.; Conard, N.J. (2015): A new approach for deciphering between single and multiple accumulation events using intra-tooth isotopic variations: Application to the Middle Pleistocene bone bed of Schöningen 13 II-4. Journal of Human Evolution 89: 114-128. doi:10.1016/j.jhevol.2015.02.012

King, C.A.M. (1971): Techniques in geomorphology. London: Arnold.

Klein, R.G.; Wolf, C.; Freeman, L.G.; Allwarden, K. (1981): The use of dental crown heights for constructing age profiles of red deer and similar species in archaeological samples. Journal of Archaeological Science 8: 1-31.

Klein, R.G.; Cruz-Uribe, K. (1983): The computation of ungulate age (mortality) profiles from dental crown heights. Paleobiology 9: 70-78.

Klein, R.G. \& Cruz-Uribe, K. (1984): The analysis of animal bones from archaeological sites. University of Chicago Press, Chicago.

Koch, P.L.; Fisher, D.C.; Dettman, D. (1989): Oxygen isotope variation in the tusks of extinct proboscideans: a measure of season of death and seasonality. Geology 17: 515-519.

Koch, P.L. (2007): Isotopic study of the biology of modern and fossil vertebrates. En Michener, R.; Lajtha, K. (ed.): Stable Isotopes in Ecology and Environtal Science. 2nd ed. Blackwell Publishing, Boston: 99-154.

Kohn, M.J. (1996): Predicting animal 8180 : accounting for diet and physiological adaptation. Geochimica et Cosmochimica Acta 60: 4811-4829.

LeCren, E. D. (1947): The determination of the age and growth of the perch (Perca fluviatilis) from the opercular bone. Journal of Animal Ecology, 16: 188-204.

Lieberman, D. E. (1994): The biological basis for seasonal increments in dental cementum and their application to archaeological research. Journal of Archaeological Science, 21: 525-539.

Lieberman, D. E.; Deacon, T. W.; Meadow, R. H. (1990): Computer image enhancement and Analysis of cementum increments as applied to teeth of Gazella gazelle. Journal of Archaeological Science, 14: 519-533.

Lubinski, P.M. (2001): Estimating age and season of death of pronghorn antelope (Antilocapra americana Ord) by means of tooth eruption and wear. International Journal of Osteoarchaeology 11: 218-230. doi:10.1002/oa.536

Luz, B.; Kolodny, Y.; Horowitz, M. (1984): Fractionation of oxygen isotopes between mammalian bone-phosphate and environmental drinking water. Geochimica et Cosmochimica Acta 48: 1689-1693.

Mannino, M.A.; Spiro, B.F.; Thomas, K.D. (2003): Sampling shells for seasonality: oxygen isotope analysis on shell carbonates of the inter-tidal gastropod Monodonta lineata (da Costa) from populations across its modern range and from a Mesolithic site in southern Britain. Journal of Archaeological Science 30: 667-679. doi:10.1016/S0305-4403(02)00238-8 
Mannino, M.A.; Thomas, K.D.; Leng, M.J.; Piperno, M.; Tusa, S.; Tagliacozzo, A. (2007): Marine resources in the Mesolithic and Neolithic at the Grotta dell'Uzzo (Sicily): evidence from isotope analyses of marine shells. Archaeometry 49: 117-133.

Mateos Cachorro, A. (2002): Apuntes sobre estacionalidad y subsistencia de los grupos humanos del Cantábrico Occidental en torno al 13000 B.P. Trabajos de Prehistoria, 59 (2): 27-41.

Mellars, P.A.; Wilkinson, M.R. (1980): Fish otoliths as evidence of seasonality in prehistoric shell middens: the evidence from Oronsay. Proceedings of the Prehistoric Society 46: 19-44

Milner, N. (1999): Pitfalls and problems in analysing and interpreting the seasonality of faunal remains. Archaeological Review from Cambridge. 51-67.

Monks, G. G. (1981): Seasonality studies. Advances in Archaeological Method and Theory, vol. 4: 177240. New York: Academic Press.

Morrison, D.; Whitridge, P. (1997): Estimating the age and sex of caribou from mandibular measurements. Journal of Archaeological Science 24: 1093-1106.

Naji, S.; Gourichon, L.; Rendu, W. (2015): La cémentochronologie. En Balasse, M.; Brugal, J.P.; Dauphin, Y.; Geigl, E.M.; Oberlin, C.; Reiche, I. (dir.): Messages d'os: Archéométrie du squelette animale et humain. Collection Sciences Archéologiques-Éditions des archives contemporaines, Paris.

O'Connor, T. (2000): The archaeology of animal bones. Sutton Publishing Ltd.

Paul A.; Mulitza S.; Pätzold J.; Wolff T. (1999): Simulation of Oxygen Isotopes in a Global Ocean Model. En Fischer G.; Wefer G. (ed.): Use of Proxies in Paleoceanography. Springer, Berlin, Heidelberg.

Payne, S. (1973): Kill-off patterns in sheep and goats: the mandibles from Aşvan Kale. Anatolian Studies 23: 281-303. doi: $10.2307 / 3642547$

Payne, S. (1987): Reference codes for wear stages in the mandibular cheek teeth of sheep and goats. Journal of Archaeological Science 14: 609-614.

Pérez Ripoll, M.; Iborra Eres, M. P.; Villaverde Bonilla, V. (2001): Aplicación del estudio de la cementocronología a materiales de los niveles magdalenienses de la Cova de Les Cendres y la Cova del Parpalló: metodología y primeros resultados. Archaeofauna 10: 113-123.

Pike-Tay, A.; Valdés, V.C.; de Quirós, F.B. (1999): Seasonal variations of the Middle-Upper Paleolithic transition at El Castillo, Cueva Morm and El Pendo (Cantabria, Spain). Journal of Human Evolution 36: $283-317$.

Polo-Díaz, A.; Benito-Calvo, A.; Martínez-Moreno, J.; Mora Torcal, R. (2016): Formation processes and stratigraphic integrity of the Middle-to-Upper Palaeolithic sequence at Cova Gran de Santa Linya (Southeastern Prepyrenees of Lleida, Iberian Peninsula). Quaternary International 417: 16-38. https://doi. org/10.1016/j.quaint.2015.10.113

Rendu, W. (2010): Hunting behavior and Neanderthal adaptability in the Late Pleistocene site of Pech-del’Azé I. Journal of Archaeological Science 37: 1798-1810. doi:10.1016/j.jas.2010.01.037

Rick, A. (1975): Bird medullary bone: a seasonal dating technique for faunal analysts. Canadian Archaeological Association, Bulletin 7: 183-190.

Rick, A. (1979): Some problems and solutions in zooarchaeological interpretation of bird bones. Paper presented at the 44th annual meeting of the Society for American Archaeology, Vancouver.

Rivals, F.; Deniaux, B. (2005): Investigation of human hunting seasonality through dental microwear analysis of two Caprinae in late Pleistocene localities in Southern France. Journal of Archaeological Science 32: 1603-1612. doi:10.1016/j.jas.2005.04.014

Rivals, F.; Solounias, N. (2007): Differences in Tooth Microwear of Populations of Caribou (Rangifer tarandus, Ruminantia, Mammalia) and Implications to Ecology, Migration, Glaciations and Dental Evolution. J. Mamm. Evol. 14: 182-192. doi:10.1007/s10914-007-9044-8

Rivals, F.; Solounias, N.; Mihlbachler, M.C. (2007): Evidence for geographic variation in the diets of late Pleistocene and early Holocene Bison in North America, and differences from the diets of recent Bison. Quaternary Research 68: 338-346. doi:10.1016/j.yqres.2007.07.012

Rivals, F.; Moncel, M.-H.; Patou-Mathis, M. (2009a): Seasonality and intra-site variation of Neanderthal occupations in the Middle Palaeolithic locality of Payre (Ardèche, France) using dental wear analyses. Journal of Archaeological Science 36: 1070-1078. doi:10.1016/j.jas.2008.12.009

Rivals, F.; Schulz, E.; Kaiser, T.M. (2009b): A new application of dental wear analyses: estimation of duration of hominid occupations in archaeological localities. Journal of Human Evolution 56: 329-339. doi:10.1016/j.jhevol.2008.11.005 
Rivals, F.; Semprebon, G.M. (2012): Paleoindian subsistence strategies and late Pleistocene paleoenvironments in the northeastern and southwestern United States: a tooth wear analysis. Journal of Archaeological Science 39: 1608-1617. doi:10.1016/j.jas.2011.12.039

Rivals, F.; Prignano, L.; Julien, M.A.; Kuitems, M.; van Kolfschoten, T.; Serangeli, J.; Drucker, D.G.; Boecherens, H.; Conard, N.J. (2014): Investigation of equid paleodiet from Schöningen 13 II-4 through dental wear and isotopic analysies: Archaeological implications. Journal of Human Evolution

Rivals, F.; Prignano, L.; Semprebon, G.M.; Lozano, S. (2015): A tool for determining duration of mortality events in archaeological assemblages using extant ungulate microwear. Scientific Reports 5, 17330

Rodríguez-Hidalgo, A.; Rivals, F.; Saladié, P.; Carbonell, E. (2016): Season of bison mortality in TD10.2 bone bed at Gran Dolina site (Atapuerca): Integrating tooth eruption, wear, and microwear methods. Journal of Archaeological Science: Reports 6: 780-789. https://doi.org/10.1016/j.jasrep.2015.11.033

Rodríguez-Hidalgo, A.; Saladié, P.; Ollé, A.; Arsuaga, J.L.; Bermúdez de Castro, J.M.; Carbonell, E. (2017): Human predatory behavior and the social implications of communal hunting based on evidence from the TD10.2 bison bone bed at Gran Dolina (Atapuerca, Spain). Journal of Human Evolution 105: 89-122. https://doi.org/10.1016/j.jhevol.2017.01.007

Rozanski, K.; Gonfiantini, R. (1990): Isótopos en estudios climatológicos. Boletín del OIEA 4/1990.

Saarinen, J.; Karme, A.; Cerling, T.; Uno, K.; Säilä, L.; Kasiki, S.; Ngene, S.; Obari, T.; Mbua, E.; Manthi, F.K.; Fortelius, M. (2015): A new tooth wear-based dietary analysis method for Proboscidea (Mammalia). Journal of Vertebrate Paleontology 35: e918546. doi:10.1080/02724634.2014.918546

Saarinen, J.; Lister, A.M. (2016): Dental mesowear reflects local vegetation and niche separation in Pleistocene proboscideans from Britain. Journal of Quaternary Science 31: 799-808. doi:10.1002/jqs.2906

Sánchez-Hernández, C.; Rivals, F.; Blasco, R.; Rosell, J. (2014): Short, but repeated Neanderthal visits to Teixoneres Cave (MIS 3, Barcelona, Spain): a combined analysis of tooth microwear patterns and seasonality. Journal of Archaeological Science 49: 317-325. doi:10.1016/j.jas.2014.06.002

Semprebon, G.M.; Godfrey, L.R.; Solounias, N.; Sutherland, M.R.; Jungers, W.L. (2004): Can low-magnification stereomicroscopy reveal diet? Journal of Human Evolution 47: 115-144. doi:10.1016/j.jhevol.2004.06.004

Sistiaga, A.; Berna, F.; Laursen, R.; Goldberg, P. (2014a). Steroidal biomarker analysis of a 14,000 years old putative human coprolite from Paisley Cave, Oregon. Journal of Archaeological Science 41: 813817. doi:10.1016/j.jas.2013.10.016

Sistiaga, A.; Mallol, C.; Galván, B.; Summons, R.E. (2014b). The Neanderthal meal: a new perspective using faecal biomarkers. PLOS ONE 9: e101045.

Solounias, N.; Semprebon, G. (2002): Advances in the Reconstruction of Ungulate Ecomorphology with Application to Early Fossil Equids. American Museum Novitates 1-49. doi:10.1206/0003-0082(2002)3 66<0001:AITROU $>2.0 . \mathrm{CO} ; 2$

Sosdian, S.; Gentry, D.K.; Lear, C.H.; Grossman, E.L.; Hicks, D.; Rosenthal, Y. (2006): Strontium to calcium ratios in the marine gastropod Conus ermineus : Growth rate effects and temperature calibration. Geochemistry, Geophysics, Geosystems 7: Q11023. doi:10.1029/2005GC001233

Spagnolo, V.; Marciani, G.; Aureli, D.; Berna, F.; Boscato, P.; Ranaldo, F.; Ronchitelli, A. (2016): Between hearths and volcanic ash: The SU 13 palimpsest of the Oscurusciuto rock shelter (Ginosa - Southern Italy): Analytical and interpretative questions. Quaternary International 417: 105-121. https://doi.or$\mathrm{g} / 10.1016 / \mathrm{j}$.quaint.2015.11.046

Spinage, C.A. (1971): Geratodontology and horn growth of the impala (Aepyceros melampus). Journal of Zoology 164, 209-225.

Spinage, C.A. (1972): Age estimation of zebra. African Journal of Ecology 10: 273-277.

Spinage, C.A. (1973): A review of the age determination of mammals by means of teeth, with especial reference to Africa. African Journal of Ecology 11: 165-187.

Spinage, C.A. (1976): Age determination of the female Grant's gazelle. African Journal of Ecology 14: $121-134$.

Stecher, H.A.; Krantz, D.E.; Lord, C.J.; Luther, G.W.; Bock, K.W. (1996): Profiles of strontium and barium in Mercenaria mercenaria and Spisula solidissima shells. Geochimica et Cosmochimica Acta 60: $3445-3456$.

Steele, T.E. (2002): Accuracy of age determinations from tooth crown heights: a test using an expanded sample of known age red deer (Cervus elaphus). En Ruscillo, D. (ed.) (2006): Recent advances in 
ageing and sexing animal bones. Proceedings of the 9th Conference of the International Council of Archaeozoology, Durham, August 2002. Oxbow Books: Oxford: 119-128.

Steele, T.E. (2004): Variation in mortality profiles of red deer (Cervus elaphus) in Middle Palaeolithic assemblages from western Europe. International Journal of Osteoarchaeology 14: 307-320. doi:10.1002/ oa. 763

Steele, T.E. (2005): Comparing methods for analysing mortality profiles in zooarchaeological and palaeontological samples. International Journal of Osteoarchaeology 15: 404-420. doi:10.1002/oa.795

Stuart-Williams, H.L.Q.; Schwarcz, H.P. (1997): Oxygen isotopic determination of climatic variation using phosphate from beaver bone, tooth enamel, and dentine. Geochimica et Cosmochimica Acta 61: 25392550.

Takesue, R.K.; van Geen, A. (2004): $\mathrm{Mg} / \mathrm{Ca}, \mathrm{Sr} / \mathrm{Ca}$, and stable isotopes in modern and Holocene Protothaca staminea shells from a northern California coastal upwelling region. Geochimica et Cosmochimica Acta 68: 3845-3861. doi:10.1016/j.gca.2004.03.021

Talbot, L.M.; Talbot, M.H. (1963): The wildebeest in Western Masailand, East Africa. Wildlife Monographs 12: 3-88.

Wang, T.; Surge, D.; Mithen, S. (2012): Seasonal temperature variability of the Neoglacial (3300-2500BP) and Roman Warm Period (2500-1600BP) reconstructed from oxygen isotope ratios of limpet shells (Patella vulgata), Northwest Scotland. Palaeogeography, Palaeoclimatology, Palaeoecology 317-318: 104-113. doi:10.1016/j.palaeo.2011.12.016

Xafis, A.; Nagel, D.; Bastl, K. (2017): Which tooth to sample? A methodological study of the utility of premolar/non-carnassial teeth in the microwear analysis of mammals. Palaeogeography, Palaeoclimatology, Palaeoecology doi:10.1016/j.palaeo.2017.09.003

Yravedra J. (2006): Tafonomía aplicada a la zooarqueología. UNED Ediciones, Madrid.

Yravedra, J.; Domínguez-Rodrigo, M.; Santonja, M.; Rubio-Jara, S.; Panera, J.; Pérez-González, A.; Uribelarrea, D.; Egeland, C.; Mabulla, A.Z.P.; Baquedano, E. (2016a): The larger mammal palimpsest from TK (Thiongo Korongo), Bed II, Olduvai Gorge, Tanzania. Quaternary International 417: 3-15. https:// doi.org/10.1016/j.quaint.2015.04.013

Yravedra, J.; Julien, M.-A.; Alcaraz-Castaño, M.; Estaca-Gómez, V.; Alcolea-González, J.; de Balbín-Behrmann, R.; Lécuyer, C.; Marcel, C.H.; Burke, A. (2016b): Not so deserted... paleoecology and human subsistence in Central Iberia (Guadalajara, Spain) around the Last Glacial Maximum. Quaternary Science Review 140: 21-38. 